\title{
Quantitative Characterization of Aberrations in X-ray Optics
}

\author{
Frank Seibotha, ${ }^{\mathrm{a},}$, Maik Kahnt ${ }^{\mathrm{a}}$, Maria Scholz ${ }^{\mathrm{a}}$, Martin Seyrich ${ }^{\mathrm{a}}$, Felix Wittwer ${ }^{\mathrm{a}}$, Jan \\ Garrevoet $^{\mathrm{a}}$, Gerald Falkenberg ${ }^{\mathrm{a}}$, Andreas Schropp ${ }^{\mathrm{a}}$, and Christian G. Schroer ${ }^{\mathrm{a}, \mathrm{c}}$ \\ ${ }^{a}$ Deutsches Elektronen-Synchrotron DESY, Notkestr. 85, 22607 Hamburg, Germany \\ ${ }^{\mathrm{b}}$ Linac Coherent Light Source, SLAC National Accelerator Laboratory, 2575 Sand Hill Road, \\ 94025 Menlo Park, CA, USA \\ ${ }^{c}$ Department Physik, Universität Hamburg, Luruper Chaussee 149, 22761 Hamburg, Germany
}

\begin{abstract}
Due to the weak interaction of X-rays with matter and their small wavelength on the atomic scale, stringent requirements are put on X-ray optics manufacturing and metrology. As a result, these optics often suffer from aberrations. Until now, X-ray optics were mainly characterized by their focal spot size and efficiency. However, both measures provide only insufficient information about optics quality. Here, we present a quantitative analysis of residual aberrations in current beryllium compound refractive lenses using ptychography followed by a determination of the wavefront error and subsequent Zernike polynomial decomposition. Known from visible light optics, we show that these measures can provide an adequate tool to determine and compare the quality of various X-ray optics.
\end{abstract}

Keywords: beam characterization, aberrations, ptychography, Zernike polynomials, hard X-ray nanobeam, compound refractive X-ray lenses

\section{INTRODUCTION}

The unique property of X-rays - to penetrate matter and their wavelength at the atomic scale - have promoted a rapid development of X-ray sources over the last century, leading to todays third generation synchrotron radiation sources and X-ray free-electron lasers (XFELs). In order to effectively make use of these highly brilliant sources suitable optics are required to focus X-rays and create small and intense beams confined to the sample region. However, it are these unique properties of X-rays that put stringent requirements on optics manufacturing and metrology. ${ }^{1}$ Thus, most X-ray optics suffer from aberrations. ${ }^{2-5}$ In order to generate a well-defined wavefront and to further advance X-ray optics fabrication a thorough characterization of created X-ray nanobeams is a prerequisite.

Over the past years, scanning coherent X-ray diffraction imaging - known as ptychography ${ }^{6,7}$ - has revolutionized not only the field of coherent X-ray imaging due to its ability to image extended objects ${ }^{8}$ with unprecedented spatial resolution ${ }^{9,10}$ and sensitivity. ${ }^{11,12}$ It also has become a viable tool to characterize X-ray beams thoroughly. ${ }^{2-4}$ The algorithm provides the capability to reconstruct the complex object transmission function as well as the complex-valued wave field illuminating the sample all at once. ${ }^{13}$ The retrieved probe function can be propagated back and forth along the optical axis using the diffraction integral of Fresnel-Kirchhoff, yielding the complete beam caustic. ${ }^{14}$ The technique can not only be applied at modern third generation synchrotron radiation sources, ${ }^{15,16}$ but also at XFELs. ${ }^{17}$

In this article, we briefly review common techniques to characterize X-ray optics and extend the method by retrieving the wave field in the exit pupil of the optics. We apply concepts and measures well known from the visible light regime, e.g. the Zernike polynomial decomposition of the wave field, root mean square and peak-tovalley wavefront error, as well as the Strehl ratio, to the field of X-ray optics. These pieces of information are not only vital to judge the quality of various X-ray optics and compare them to one another, but also provides invaluable information to improve current optical systems.

Further author information: (Send correspondence to Frank Seiboth)

Frank Seiboth: E-mail: frank.seiboth@desy.de, Telephone: +1 (650) 926-2048

Advances in X-Ray/EUV Optics and Components XI, edited by Ali M. Khounsary, Shunji Goto, Christian Morawe, Proc. of SPIE Vol. 9963, 99630P - (C) 2016 SPIE · CCC code: 0277-786X/16/\$18 · doi: 10.1117/12.2237646 


\section{BASIC WAVE FIELD CHARACTERIZATION USING PTYCHOGRAPHY}

We characterized a nanofocusing optics consisting of 20 beryllium compound refractive lenses (Be CRLs) with a radius of curvature $R=50 \mu \mathrm{m}$ and a geometrical aperture of $D=300 \mu \mathrm{m}$. The experiment was carried out at the microprobe branch of beamline P06 of PETRA III at DESY. The X-ray beam was monochromatized to a photon energy of $E=8.2 \mathrm{keV}$ using a Si-111 monochromator. The full-width-at-half-maximum (FWHM) X-ray source size $S$ of the undulator is $82 \times 14 \mu^{2}$ in the horizontal and vertical direction, respectively. In order to make use of a beam characterization by ptychography a coherent illumination of the optics is required, meaning a transverse coherence length $\zeta_{t} \gtrsim D$. With

$$
\zeta_{t}=\frac{4 \ln 2}{\pi} \frac{\lambda L}{S}
$$

and a distance $L=92 \mathrm{~m}$ of the experiment from the undulator source we achieve a transverse coherence length of $75 \times 440 \mathrm{\mu m}^{2}$, which is insufficient in horizontal direction. In order to increase the coherence properties at the experiment we used a single prefocusing Be CRL $(R=50 \mu \mathrm{m}, D=400 \mu \mathrm{m})$ at a distance of $43.5 \mathrm{~m}$ downstream of the undulator source. This creates a secondary source with a FWHM size of $11 \times 1.8 \mathrm{um}^{2}$ at $49 \mathrm{~m}$, providing a sufficient transverse coherence length of $260 \times 1600 \mathrm{\mu m}^{2}$ at the experiment. The aperture of the optics was defined by two pinholes with a diameter of $300 \mu \mathrm{m}$ at the entrance and exit of the Be CRL stack.

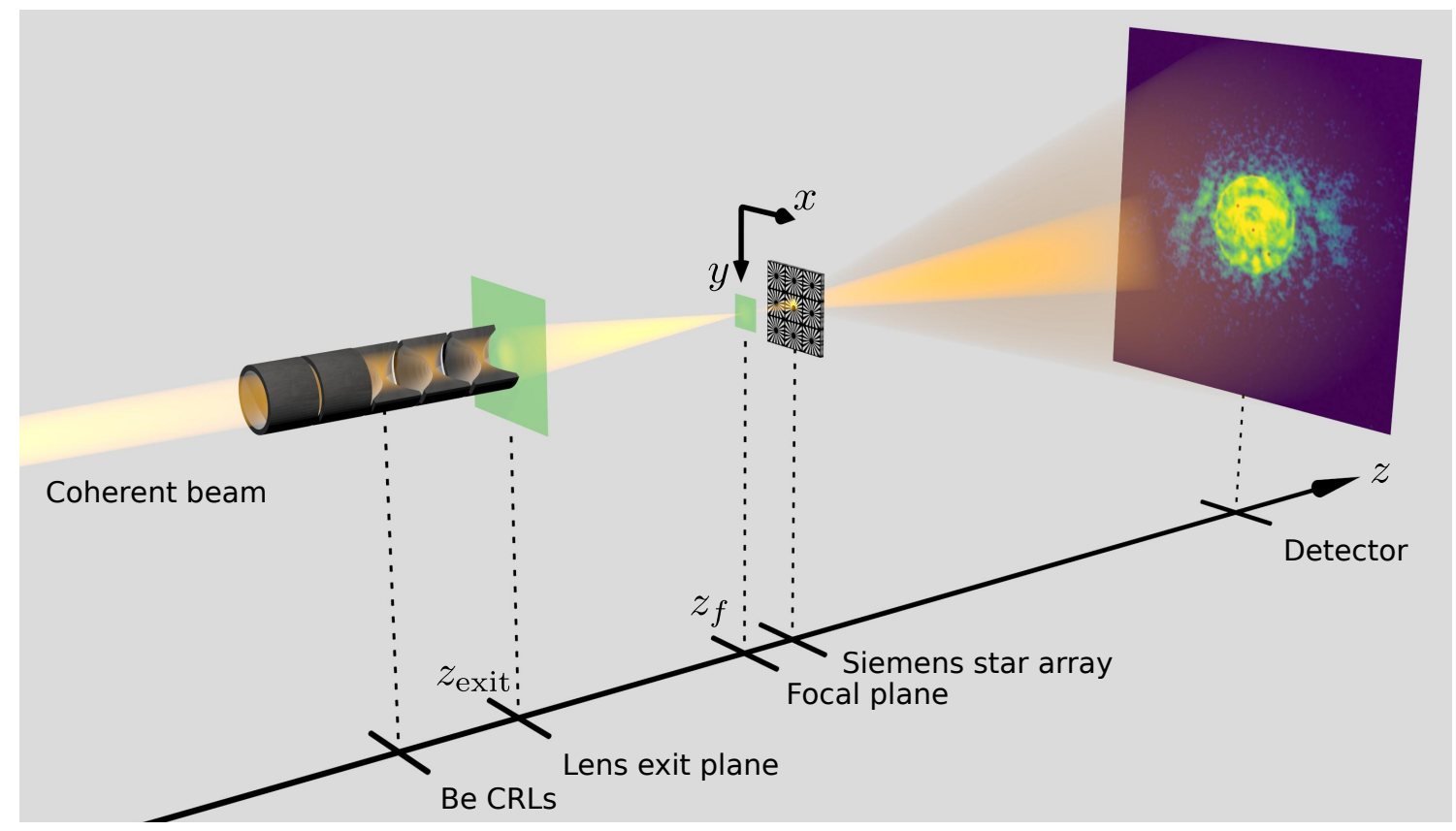

Figure 1. Schematic of the ptychographic setup to determine at wavelength residual aberrations in a stack of 20 Be CRLs. The sample, a Siemens star array, is moved perpendicular to the beam on an $x-y$ grid. Diffraction patterns are recorded in the far field at each scan position. The algorithm reconstructs the complex-valued probe function that can be propagated to the focal plane $z_{f}$ and to the exit pupil of the optics located at $z_{\text {exit }}$ for further analysis.

To characterize the divergent beam we used a strongly scattering test sample structured into $1 \mu \mathrm{m}$ thick tungsten on a diamond substrate. The pattern is an array of 20 by 20 small Siemens stars each measuring $2 \times 2 \mathrm{\mu m}^{2}$ with smallest features of $50 \mathrm{~nm}$. The test pattern is placed in the vicinity of the focal plane as shown in Fig. 1. The sample is scanned transversely to the beam in $x$ and $y$ direction in a grid fashion with a step size of $200 \mathrm{~nm}$. We recorded $21 \times 21$ diffraction patterns with a DECTRIS Eiger X4M, ${ }^{18}$ covering an area of $4 \times 4 \mathrm{~mm}^{2}$ on the sample. Our ptychography algorithm is based on the ePIE ${ }^{7}$ and enhanced by a position refinement. ${ }^{19}$ With the reconstructed complex-valued wave field and using the Fresnel-Kirchhoff diffraction integral the complete beam caustic as shown in Fig. 2a) can be reconstructed. The caustic shows a clear signature of spherical aberrations, manifesting itself in paraxial rays focused further upstream (arrow in Fig. 2a) than rays originating 
from larger angles focused into the main focal plane. A slice through the focal plane, which we determined by the highest peak intensity, is marked by the dashed line in Fig. 2a) and shown in Fig. 2b). The central focal spot is surrounded by strong side lobes, which are enhanced in intensity owing to the spherical aberration.

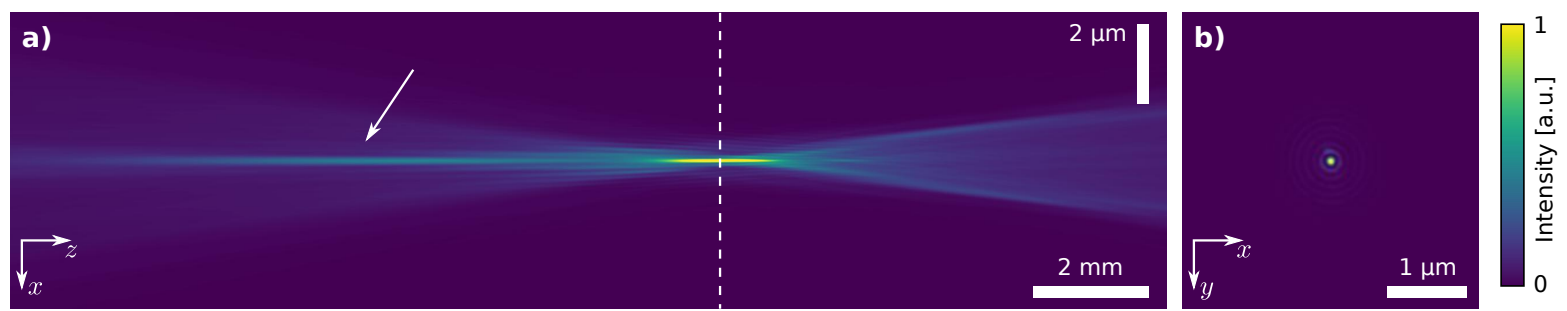

Figure 2. a) A beam caustic can be reconstructed when the probe function is propagated along the optical axis. The plane with the highest intensity, assumed to be the focal plane of the optics, is marked by the dashed line. The arrow highlights paraxial rays being focused further upstream. b) Intensity distribution in the focal plane, as marked by the dashed line in a).

Often the focal spot size of the nanofocusing optics is used as a measure to judge their quality. A line profile in the $x$ direction through the focal plane from Fig. 2b) is shown in Fig. 3 together with a line profile obtained from modeling the same stack of Be CRLs with a perfect shape. For this we numerically propagated a flat beam through the lens stack, treating every single lens as a thin lens. To compare the profiles quantitatively the two-dimensional intensity distributions in the focal plane were normalized, so that both optics had the same total intensity. Fig. 3a) clearly shows a significant difference in the magnitude of the central speckle. The strong decrease is caused by enhanced side lobes, as shown more clearly on a logarithmic scale in Fig. 3b). However, the determined FWHM focus size of 142.6(12) nm and 143(3) nm for the measured and modeled lens, respectively, shows no deviation. Since the size of the smallest speckle in the focal plane only depends on the numerical aperture $(N A)$ of the optics, which is the same in both cases, this is not surprising. While the speckle size is a great measure to showcase the achieved $N A$ of the optical system, other measures are needed to describe the aberrations of X-ray optics.
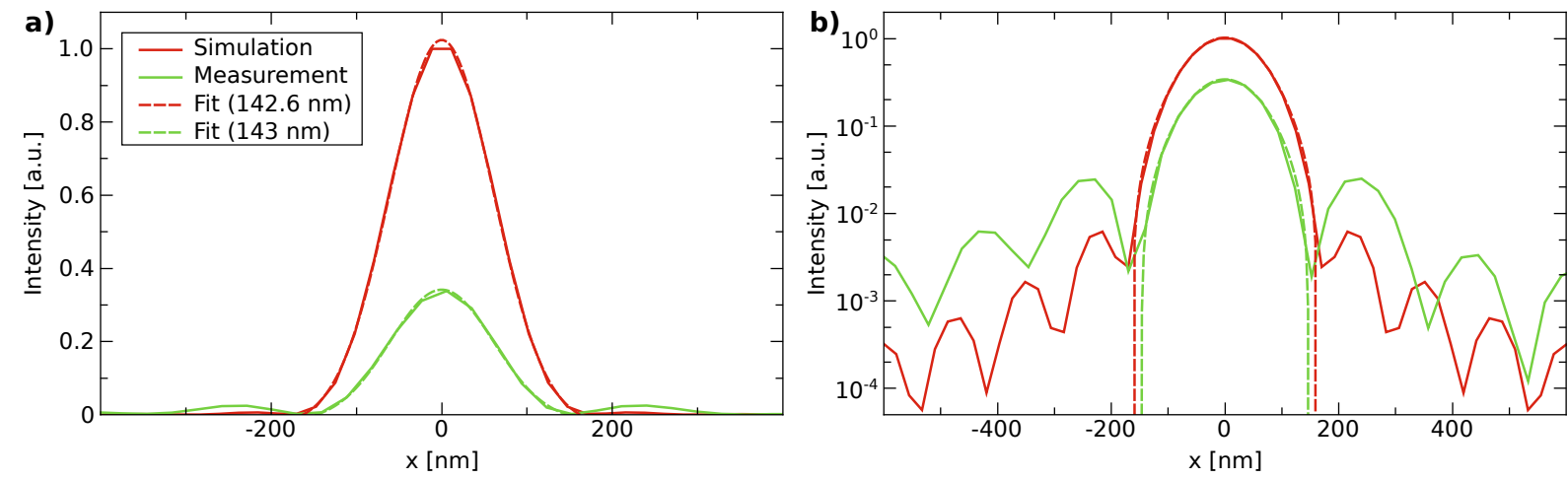

Figure 3. a) Line profile of the intensity distribution in the focal plane in horizontal direction taken from Fig. 2b). b) Line profile from a) on a logarithmic scale to highlight the contribution of side lobes.

Known from the visible light regime, the Strehl ratio is used often to describe the quality of a given optics by a single measure. ${ }^{20,21}$ It is defined as the ratio of the peak intensity in the focal plane, or alternatively the intensity on the optical axis, between an optical system and the maximum attainable intensity of an ideal optics. Here, we will use the peak intensity in the focal plane, as obtained by the Gaussian fit in Fig. 3. With this we obtain a Strehl ratio for the stack of Be CRLs of 0.35. An optics is considered to be diffraction-limited, if the root mean square (RMS) phase error of the wavefront is 14 times less than its wavelength, also know as the Maréchal criterion. ${ }^{22}$ This leads to a Strehl ratio of 0.8 for a reference sphere with a wavefront departure 
of $\lambda / 14 .{ }^{23}$ A perfect system would show a Strehl ratio of 1 . Thus, the characterized Be CRL stack is far from being considered diffraction limited.

In the next section we use the retrieved complex-valued wave field for an in-depth characterization of present aberrations. While the Strehl ratio is a great measure for the overall quality of the optical system, it gives no insight on the specific type of wavefront deformation. Therefore, we will evaluate the wave field at the exit pupil of the optics and calculate the RMS phase error of the wavefront and identify the magnitude of primary aberrations by a Zernike polynomial decomposition of the wave field.

\section{CHARACTERIZING ABERRATIONS}
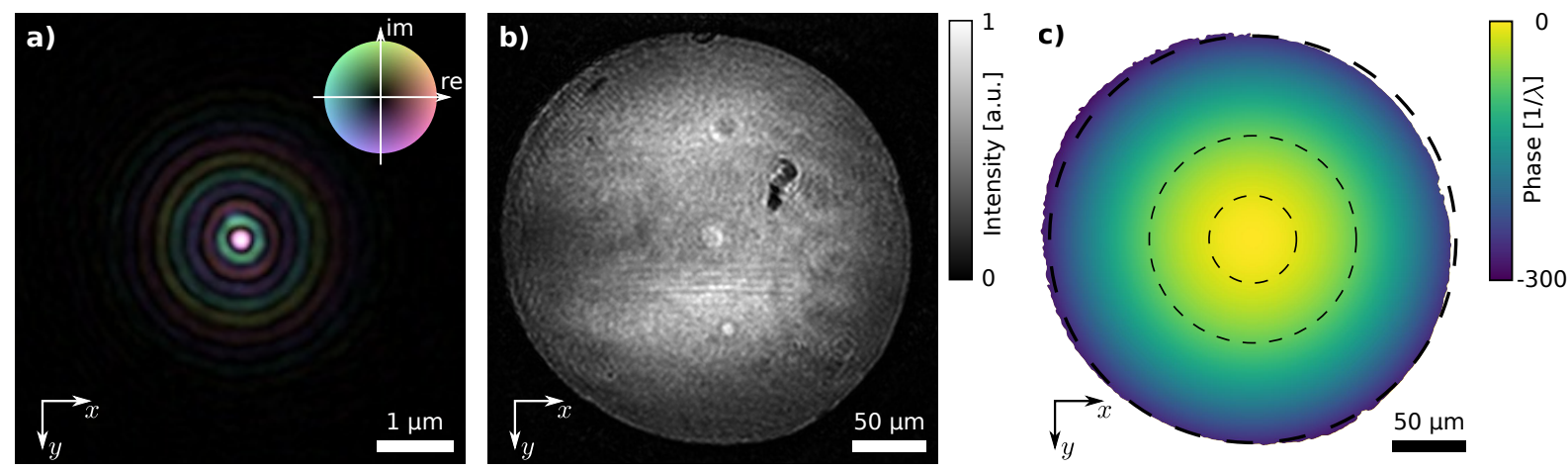

Figure 4. a) Complex-valued wave field in the focal plane $z_{f}$ as shown in Fig. 1. b) Intensity distribution at the exit plane $z_{\text {exit }}$ of the Be CRL stack after backpropagating a) by a distance $\Delta z$. c) Unwrapped phase distribution at the exit pupil $z_{\text {exit. }}$. The dashed concentric circles highlight the center of the spherical wave.

Until now, we evaluated the wave field retrieved from ptychography mainly in the vicinity of the focal plane by calculating the beam caustic as shown in Fig. 2a) that already revealed spherical aberrations on a qualitative basis. To make use of the full potential of the reconstructed complex-valued wave field in the focal plane (cf. Fig. 4a) we will propagate the field from $z_{f}$ by $\Delta z$ back to the exit pupil of the Be CRL stack at position $z_{\text {exit }}$ (cf. Fig. 1). The obtained intensity distribution and unwrapped phase are shown in Figs. 4b) and 4c), respectively. The aperture in Fig. 4b) is not completely round due to manufacturing tolerances in the two pinholes defining the geometrical aperture. In addition a small tilt of the whole lens stack due to alignment errors may clip the aperture. The dark spot is likely originating from contamination of the lens stack with dust. In the phase distribution shown in Fig. 4c) the center of the spherical wave is highlighted by the dashed circles, indicating that the aperture is slightly off-axis.

To uncover slight wavefront deviations we subtracted the phase $\varphi_{\Delta z}(x, y)$ of a perfect spherical wave

$$
\varphi_{\Delta z}(x, y)=-k\left(\sqrt{\Delta z^{2}+x^{2}+y^{2}}-\Delta z\right)
$$

from the phase distribution shown in Fig. 4c). $\Delta z$ is an initial guess of the radius of curvature being equal to the propagation distance from the estimated focal plane $z_{f}$ to exit pupil of the lens $z_{\text {exit }}$ (cf. Fig. 1). Due to the rotational symmetry of the Be CRLs and their weak absorption the aperture is dominated by the geometry of the lens and can be best described by a disk. To adequately describe aberrations of a circular pupil, Zernike polynomials ${ }^{24}$ are commonly used. We can expand any smooth function $f(r, \phi)$ on the unit disk $(r \in[0,1], \phi \in$ $[0,2 \pi[)$ into a set of base functions

$$
f(r, \phi)=\sum_{n=1}^{\infty} \sum_{m=-n}^{n} z_{n}^{m} V_{n}^{m}(r, \phi) .
$$

We call $z_{n}^{m}$ the Zernike coefficients and $V_{n}^{m}(r, \phi)$ are the complex Zernike polynomials defined as $V_{n}^{m}(r, \phi)=$ $R_{n}^{m}(r) \exp (\mathrm{i} m \phi)$. The degree of the radial polynomial is denoted by $n$ and the azimuthal dependence is represented by $m$. To describe wavefront deformations, which are real valued scalar functions, we define the real-valued 
Zernike polynomials

$$
\begin{gathered}
Z_{n}^{m}=\frac{1}{2}\left(V_{n}^{m}+V_{n}^{-m}\right)=R_{n}^{m}(r) \cos (m \phi), \\
Z_{n}^{-m}=\frac{1}{2 \mathrm{i}}\left(V_{n}^{m}-V_{n}^{-m}\right)=R_{n}^{m}(r) \sin (m \phi), m \neq 0 .
\end{gathered}
$$

With this orthogonal set of scalar polynomials aberrations on a circular pupil can be described. For optics with annular pupils or square apertures other base functions exist. ${ }^{25,26}$

After the initial subtraction of $\varphi_{\Delta z}$ we fitted the first four Zernike polynomials $Z_{0}^{0}, Z_{1}^{-1}, Z_{1}^{1}$, and $Z_{2}^{0}$ (representing phase offset, tilt in $x$, tilt in $y$, and defocus) to the remaining phase error to compensate for a slight tilt due to alignment inaccuracies, and to fit the correct wavefront curvature. The unit disk for the Zernike polynomials was chosen as shown by the outer dashed circle in Fig. 4c). When subtracting the Zernike wavefront representation from the phase error at the exit pupil we retrieve the residual wavefront deformation of the optics as shown in Fig. 5a). The contamination on the lens also leads to additional features in the phase distribution, highlighted by the dashed ellipse for example. Other small features can also be seen on the lower right part of the aperture. These impurities are also faintly visible in the intensity distribution shown in Fig. 4b).
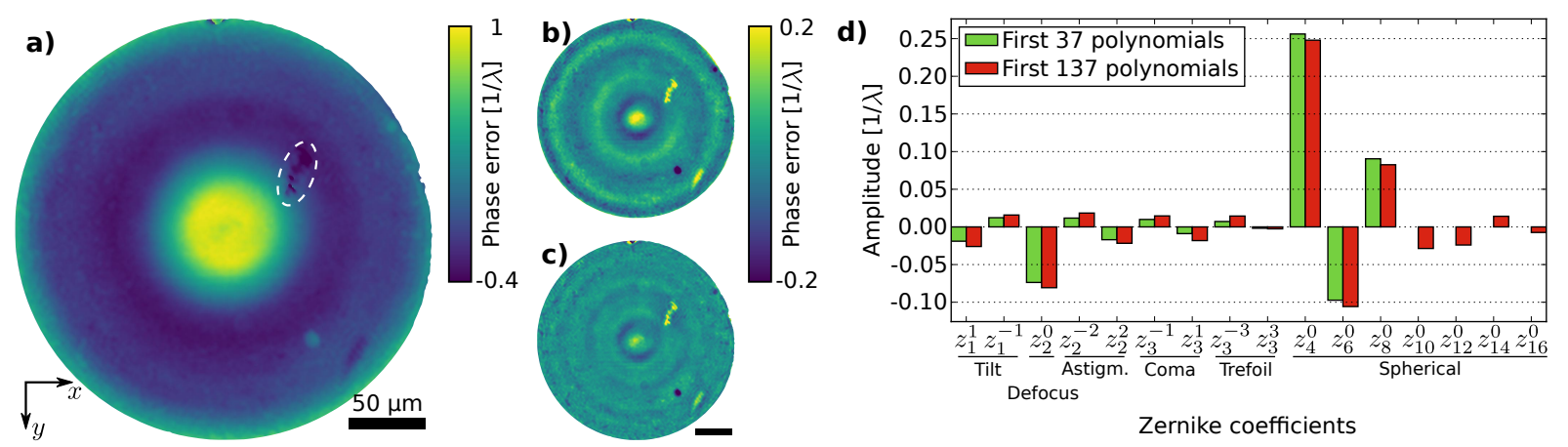

Figure 5. a) Residual aberrations of the Be CRLs after fitting the first 4 Zernike polynomials $Z_{0}^{0}, Z_{1}^{-1}, Z_{1}^{1}$, and $Z_{2}^{0}$ that compensate for slight misalignment only. b),c) Remaining wavefront error after fitting the first 37 or 137 polynomials, respectively. d) Strength of various aberrations present in the Be CRLs represented by the amplitude of Zernike coefficients. The phase offset $Z_{0}^{0}$ and higher order non-spherical aberrations are omitted in the plot due to minor contributions but were considered for fitting.

To quantify the strength of aberrations one can determine the RMS phase error in Fig. 5a) and also the peak-to-valley (PV) phase error. Values are presented in Table 1. The PV error reduces when the contaminated regions are masked. However, the RMS phase error stays nearly a constant. While the PV error gives a simple measure of the maximum departure from an ideal wavefront, the measure might give misleading information on optics quality. Since the area over which the error occurs is not taken into account, small impurities can increase the value dramatically, without much effect on actual beam quality. Here, the RMS error provides a much better value to quantify aberrations.

From Fig. 5a) it is already apparent that spherical aberration is the most dominant primary aberration of the investigated Be CRLs. To measure the contribution of individual types of aberrations, taking astigmatism, coma, spherical aberration, and higher order ones into account, the first 37 Zernike polynomials $Z_{0}^{0}-Z_{8}^{0}$ were fitted to the wavefront. The remaining phase error and the magnitude of individual Zernike coefficients is shown in Figs. 5b) and 5d), respectively. The polynomial decomposition shows significant contributions only from the 0th-order azimuthal polynomials $Z_{2}^{0}, Z_{4}^{0}, Z_{6}^{0}$, and $Z_{8}^{0}$. These represent defocus, spherical aberration, and higher order spherical terms. The dominant aberration is the primary spherical aberration $Z_{4}^{0}$ with an amplitude of $0.26 \lambda^{-1}$. Higher order azimuthal aberrations such as tilt, astigmatism, coma, and trefoil are present, but their strength is negligible.

The fit error shown in Fig. 5b) shows a clear sign of remaining spherical contributions not covered by the considered polynomials. In an attempt to increase the fit quality we used up to 137 Zernike polynomials to 
represent the wavefront more accurately. The remaining phase error is displayed in Fig. 5c) and the coefficients are shown in Fig. 5d). The fit improved from a standard deviation of $\sigma_{37}=0.045 \lambda^{-1}$ to $\sigma_{137}=0.025 \lambda^{-1}$ when going from 37 to 137 Zernike polynomials. In this way, the spherical deformation of the Be CRLs is well represented. Despite the need for higher orders to be considered in this case, Zernike polynomials provide a convenient base set to characterize X-ray optics. Their lower orders tightly represent Seidel aberrations, which lead to typical imaging errors.

Table 1. Measures to describe the wavefront deformation caused by the investigated Be CRL stack shown in Fig. 5a).

\begin{tabular}{|l|l|l|}
\hline & unmasked & impurities masked \\
\hline root-mean-square phase error $\Delta \varphi_{\mathrm{RMS}}\left[\lambda^{-1}\right]$ & 0.29 & 0.29 \\
\hline peak-to-valley phase error $\Delta \varphi_{\mathrm{PV}}\left[\lambda^{-1}\right]$ & 1.58 & 1.32 \\
\hline
\end{tabular}

\section{CONCLUSIONS}

Ptychography has become an invaluable tool to characterize X-ray beams. With the combination of techniques well known from the visible light regime we have demonstrated several possibilities to quantitatively describe the quality of X-ray optics that go beyond current methods in the X-ray regime:

- The often used focal spot size provides a good measure to determine the achieved $N A$ of the optics, but may not give much insight into present aberrations. We show that the Strehl ratio provides a convenient way to additionally describe the quality of the focusing optics by comparing the measured peak intensity in the focal plane to numerical models of the optics.

- A propagation of the complex-valued wave field from the vicinity of the focal plane back to the exit pupil of the optics gives access to a highly resolved and very sensitive map of wavefront deformations. This information can be used to determine the general magnitude of phase errors or to quantitatively identify the strength of Seidel aberrations by a Zernike polynomial decomposition.

- The metrology provided by these techniques can be used to model aberrations in X-ray optics and to identify manufacturing errors in great detail.

As most X-ray optics suffer from imperfections due to manufacturing limitations the demonstrated methods provide excellent measures to assess the performance of various X-ray optics and to further refine their quality.

\section{ACKNOWLEDGMENTS}

The authors thank Martin Döring and Dirk Samberg for their technical support and Ulrich Vogt for providing the test sample. F.S. acknowledges a Peter Paul Ewald fellowship from the Volkswagen foundation. This work is supported by the German Ministry of Education and Research (BMBF) under grant number 05K13OD2. Beamtime at PETRA III was granted within the user program.

\section{REFERENCES}

[1] Yumoto, H., Mimura, H., Koyama, T., Matsuyama, S., Tono, K., Togashi, T., Inubushi, Y., Sato, T., Tanaka, T., Kimura, T., Yokoyama, H., Kim, J., Sano, Y., Hachisu, Y., Yabashi, M., Ohashi, H., Ohmori, H., Ishikawa, T., and Yamauchi, K., "Focusing of x-ray free-electron laser pulses with reflective optics," Nat Photon 7, 43-47 (2013).

[2] Kewish, C. M., Guizar-Sicairos, M., Liu, C., Qian, J., Shi, B., Benson, C., Khounsary, A. M., VilaComamala, J., Bunk, O., Fienup, J. R., Macrander, A. T., and Assoufid, L., "Reconstruction of an astigmatic hard x-ray beam and alignment of k-b mirrors from ptychographic coherent diffraction data," Opt. Express 18, 23420-23427 (2010). 
[3] Schropp, A., Boye, P., Feldkamp, J. M., Hoppe, R., Patommel, J., Samberg, D., Stephan, S., Giewekemeyer, K., Wilke, R. N., Salditt, T., Gulden, J., Mancuso, A. P., Vartanyants, I. A., Weckert, E., Schoder, S., Burghammer, M., and Schroer, C. G., "Hard x-ray nanobeam characterization by coherent diffraction microscopy," Applied Physics Letters 96(9), 091102 (2010).

[4] Vila-Comamala, J., Diaz, A., Guizar-Sicairos, M., Mantion, A., Kewish, C. M., Menzel, A., Bunk, O., and David, C., "Characterization of high-resolution diffractive x-ray optics by ptychographic coherent diffractive imaging," Opt. Express 19, 21333-21344 (2011).

[5] Nilsson, D., Uhlén, F., Holmberg, A., Hertz, H. M., Schropp, A., Patommel, J., Hoppe, R., Seiboth, F., Meier, V., Schroer, C. G., Galtier, E., Nagler, B., Lee, H. J., and Vogt, U., "Ronchi test for characterization of nanofocusing optics at a hard x-ray free-electron laser," Opt. Lett. 37, 5046-5048 (2012).

[6] Thibault, P., Dierolf, M., Menzel, A., Bunk, O., David, C., and Pfeiffer, F., "High-resolution scanning x-ray diffraction microscopy," Science 321(5887), 379-382 (2008).

[7] Maiden, A. M. and Rodenburg, J. M., "An improved ptychographical phase retrieval algorithm for diffractive imaging," Ultramicroscopy 109(10), 1256 - 1262 (2009).

[8] Rodenburg, J., Hurst, A., and Cullis, A., "Transmission microscopy without lenses for objects of unlimited size," Ultramicroscopy 107(2-3), 227 - 231 (2007).

[9] Takahashi, Y., Suzuki, A., Zettsu, N., Kohmura, Y., Senba, Y., Ohashi, H., Yamauchi, K., and Ishikawa, T., "Towards high-resolution ptychographic x-ray diffraction microscopy," Phys. Rev. B 83, 214109 (2011).

[10] Schropp, A., Hoppe, R., Patommel, J., Samberg, D., Seiboth, F., Stephan, S., Wellenreuther, G., Falkenberg, G., and Schroer, C. G., "Hard x-ray scanning microscopy with coherent radiation: Beyond the resolution of conventional x-ray microscopes," Applied Physics Letters 100(25), 253112 (2012).

[11] Hoppe, R., Reinhardt, J., Hofmann, G., Patommel, J., Grunwaldt, J.-D., Damsgaard, C. D., Wellenreuther, G., Falkenberg, G., and Schroer, C. G., "High-resolution chemical imaging of gold nanoparticles using hard x-ray ptychography," Applied Physics Letters 102(20), 203104 (2013).

[12] Takahashi, Y., Suzuki, A., Furutaku, S., Yamauchi, K., Kohmura, Y., and Ishikawa, T., "High-resolution and high-sensitivity phase-contrast imaging by focused hard x-ray ptychography with a spatial filter," Applied Physics Letters 102(9), 094102 (2013).

[13] Thibault, P., Dierolf, M., Bunk, O., Menzel, A., and Pfeiffer, F., "Probe retrieval in ptychographic coherent diffractive imaging," Ultramicroscopy 109(4), 338 - 343 (2008).

[14] Hönig, S., Hoppe, R., Patommel, J., Schropp, A., Stephan, S., Schöder, S., Burghammer, M., and Schroer, C. G., "Full optical characterization of coherent x-ray nanobeams by ptychographic imaging," Opt. Express 19, 16324-16329 (2011).

[15] Kewish, C. M., Thibault, P., Dierolf, M., Bunk, O., Menzel, A., Vila-Comamala, J., Jefimovs, K., and Pfeiffer, F., "Ptychographic characterization of the wavefield in the focus of reflective hard x-ray optics," Ultramicroscopy 110(4), 325 - 329 (2010).

[16] Schroer, C. G., Brack, F.-E., Brendler, R., Hönig, S., Hoppe, R., Patommel, J., Ritter, S., Scholz, M., Schropp, A., Seiboth, F., Nilsson, D., Rahomäki, J., Uhlén, F., Vogt, U., Reinhardt, J., and Falkenberg, G., "Hard x-ray nanofocusing with refractive x-ray optics: full beam characterization by ptychographic imaging," Proc. SPIE 8848, 884807 (2013).

[17] Schropp, A., Hoppe, R., Patommel, J., Seiboth, F., Uhlén, F., Vogt, U., Lee, H. J., Nagler, B., Galtier, E. C., Zastrau, U., Arnold, B., Heimann, P., Hastings, J. B., and Schroer, C. G., "Scanning coherent x-ray microscopy as a tool for xfel nanobeam characterization," Proc. SPIE 8849, 88490R (2013).

[18] Johnson, I., Bergamaschi, A., Billich, H., Cartier, S., Dinapoli, R., Greiffenberg, D., Guizar-Sicairos, M., Henrich, B., Jungmann, J., Mezza, D., Mozzanica, A., Schmitt, B., Shi, X., and Tinti, G., "Eiger: a single-photon counting x-ray detector," Journal of Instrumentation 9(05), C05032 (2014).

[19] Hoppe, R., Meier, V., Patommel, J., Seiboth, F., Lee, H. J., Nagler, B., Galtier, E. C., Arnold, B., Zastrau, U., Hastings, J., Nilsson, D., Uhlén, F., Voigt, U., Hertz, H. M., Schroer, C. G., and Schropp, A., "Full characterization of a focused wave field with sub $100 \mathrm{~nm}$ resolution," Proc. SPIE 8778, 87780G (2013).

[20] Mahajan, V. N., "Strehl ratio for primary aberrations: some analytical results for circular and annular pupils," J. Opt. Soc. Am. 72, 1258-1266 (1982). 
[21] Perevezentsev, E., Poteomkin, A., and Khazanov, E., "Comparison of phase-aberrated laser beam quality criteria," Appl. Opt. 46, 774-784 (2007).

[22] Maréchal, A., "Étude des effets combinés de la diffraction et des aberrations géométriques sur limage dun point lumineux," Rev. Opt 26, 257-277 (1947).

[23] Born, M. and Wolf, E., [Principles of optics: electromagnetic theory of propagation, interference and diffraction of light], Pergamon Press (1980).

[24] von F. Zernike, "Beugungstheorie des schneidenver-fahrens und seiner verbesserten form, der phasenkontrastmethode," Physica 1(7), 689 - 704 (1934).

[25] Swantner, W. and Chow, W. W., "Gram-schmidt orthonormalization of zernike polynomials for general aperture shapes," Appl. Opt. 33, 1832-1837 (1994).

[26] Ye, J., Gao, Z., Wang, S., Cheng, J., Wang, W., and Sun, W., "Comparative assessment of orthogonal polynomials for wavefront reconstruction over the square aperture," J. Opt. Soc. Am. A 31, 2304-2311 (2014). 